\title{
Метрологическое обеспечение измерений временных характеристик импульсного лазерного излучения в пикосекундном диапазоне
}

М. В. Канзюба

ФГУП «ВНИИОФИ», www. vniiofi.ru; mkanzyuba@vniiofi.ru; Москва, Россия

Прецизионная обработка материалов, техника лазерной дальнометрии и медицины используют пикосекундные импульсные лазерные системы. Это требует создания средств измерений временных характеристик импульсного лазерного излучения. В статье представлены результаты деятельности ВНИИОФИ в области метрологического обеспечения измерений временных характеристик импульсного лазерного излучения в пикосекундном диапазоне.

Ключевые слова: метрологическое обеспечение, импульсное лазерное излучение, временные характеристики

\section{Metrological Support of Measurements of Temporal Parameters of Pulsed Laser Radiation in The Picosecond Range}

\section{M.V. Kanzyuba}

The All-Russian Research Institute for Optical and Physical Measurements,www.vniiofi.ru; mkanzyuba@vniiofi.ru; Moscow, Russia

\begin{abstract}
Precision material processing, laser ranging and medicine techniques use picosecond pulsed laser systems. This requires the creation of measuring instruments for the temporal characteristics of pulsed laser radiation. Presented in the article are the results of activities of the VNIIOFI in the field of metrological support of measurements of temporal parameters of pulsed laser radiation in the picosecond range.
\end{abstract}

Keywords: metrological support, pulsed laser radiation, temporal parameters 


\section{ВВЕДЕНИЕ}

В настоящее время в таких областях, как прецизионная обработка материалов, лазерная дальнометрия, медицина, научные исследования, оборонная техника, получают все более широкое распространение пикосекундные импульсные лазерные системы. Свойства таких систем существенным образом зависят от временных характеристик генерируемого импульсного излучения. В связи с этим возрастает потребность в средствах измерений (СИ) временных характеристик импульсного лазерного излучения (в частности, длительности импульсов, интервалов между ними) в пикосекундном диапазоне $\left(10^{-12}-10^{-9}\right.$ с). Для нормирования метрологических характеристик СИ необходимо наличие метрологического обеспечения - эталонной базы, поверочной схемы, методик поверки и испытаний в целях утверждения типа СИ.

Федеральным агентством по техническому регулированию и метрологии (Росстандарт) на Всероссийский научно-исследовательский институт оптико-физических измерений (ВНИИОФИ) возложены функции головной организации в области оптико-физических измерений и метрологии быстропротекающих процессов. Измерения временных характеристик пикосекундных оптических импульсов относятся к обеим указанным категориям.

Обычно для прямых измерений длительности оптических импульсов в наносекундном диапазоне используется высокоскоростной фотодетектор, подключенный к широкополосному осциллографу. Чтобы иметь возможность измерять пикосекундные импульсы, такая система должна иметь очень малое время нарастания переходной характеристики (ПХ). Существуют осциллографы, которые имеют время нарастания ПХ не более 4 пс [1], но в этом случае ограничивающим фактором является время нарастания ПХ фотодетектора, которое пока не превосходит 7 пс для лучших образцов [2]. Использование стробоскопических осциллографов возможно только для повторяющихся сигналов с хорошо воспроизводящейся формой.

Таким образом, задача измерения временных характеристик лазерных импульсов в пикосекундном диапазоне предъявляет высокие требования к быстродействию аппаратуры (полоса пропускания, время нарастания ПХ). Кроме того, необходимо учитывать высокую стоимость и ограниченную доступность такой аппаратуры. В то же время существует альтернатива - фотохронографические электроннооптические камеры (ЭОК), также известные как стрик-камеры (англ. streak camera). ЭОК пригодны для исследования однократных сигналов и обладают временным разрешением вплоть до 0,1 пс [3]. Принцип действия ЭОК основан на преобразовании потока оптического излучения, прошедшего через входную щель на фотокатод электронно-оптического преобразователя (ЭОП), в поток фотоэлектронов, который отклоняется (развертывается) импульсным электрическим полем в направлении, перпендикулярном щели, и снова преобразуется в световой поток в люминофоре, нанесенном на волоконнооптическую пластину (ВОП). Развернутое по люминесцентному экрану эОП изображение входной щели, яркость которого пропорциональна мгновенной мощности излучения, регистрируется с помощью ПЗС-матрицы. По этому изображению можно определять форму оптических импульсов и измерять их временные характеристики.

ВНИИОФИ занимается разработкой и производством ЭОК на протяжении всей своей истории. Так, первая в мире промышленная ЭОК ФЭР-2 была разработана во ВНИИОФИ в 1966 году. [4]. Поэтому во ВНИИОФИ при разработке аппаратуры для измерений временных характеристик импульсного лазерного излучения в пикосекундном диапазоне ставка сделана на использование ЭОК собственного производства.

Далее представлены результаты деятельности ВНИиОФИ в области метрологического обеспечения измерений временных характеристик импульсного лазерного излучения в пикосекундном диапазоне.

1. Создание и включение в состав усовершенствованного Государственного первичного специального эталона ГЭТ 187-2016 комплекса технических средств для воспроизведения, хранения и передачи единицы длительности импульса лазерного излучения в диапазоне от $5 \cdot 10^{-11}$ до $1 \cdot 10^{-9} \mathrm{C}$

С 2010 года во ВНИИОФИ эксплуатируется Государственный первичный специальный эталон единиц энергии, распределения плотности энергии, длительности импульса и длины волны лазерного излучения ГЭТ 187-2010 [5], в состав которого входит комплекс средств измерений для воспроизведения и передачи единицы длительности импульса лазерного излучения. Данный комплекс воспроизводит единицу длительности импульса в диапазоне от $1 \cdot 10^{-9}$ до $1 \cdot 10^{-6} \mathrm{c}$.

В результате выполнения работы по совершенствованию эталона создан и включен в его состав новый комплекс технических средств для воспроизведения, хранения и передачи единицы длительности импульса лазерного излучения в диапазоне 


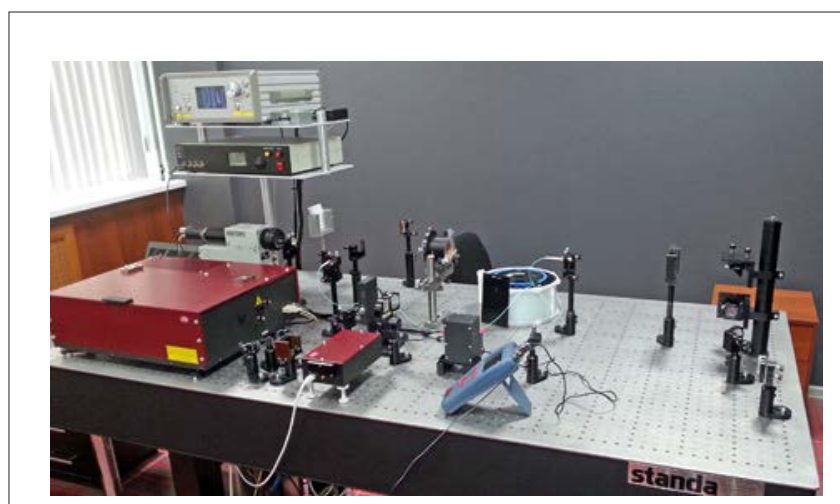

Puс. 1. Внешний вид комплекса технических средств для воспроизведения, хранения и передачи единицы диительности импульса лазерного излучения в диапазоне от $5 \cdot 10^{-11}$ до $1 \cdot 10^{-9}$ с в составе Государственного первичного специального эталона ГЭТ 187-2016

от $5 \cdot 10^{-11}$ до $1 \cdot 10^{-9}$ с. Принцип действия комплекса основан на измерении длительности импульса, генерируемого лазерной системой, с помощью ЭОК, временная шкала которой калибруется с использованием интерферометра Фабри-Перо. Подробное описание устройства и работы комплекса дано в публикации [6]. Основные характеристики комплекса представлены в табл. 1, внешний вид комплекса показан на рис. 1.

Усовершенствованный Государственный первичный специальный эталон ГЭТ 187-2016 утвержден приказом Росстандарта № 2089 от 30.12.2016.

2. Создание и аттестация Государственного вторичного эталона единицы длительности импульса лазерного излучения в диапазоне от $3 \cdot 10^{-12}$ до $1 \cdot 10^{-9} \mathrm{c}$ По принципу действия вторичный эталон аналогичен рассмотренному выше комплексу в составе

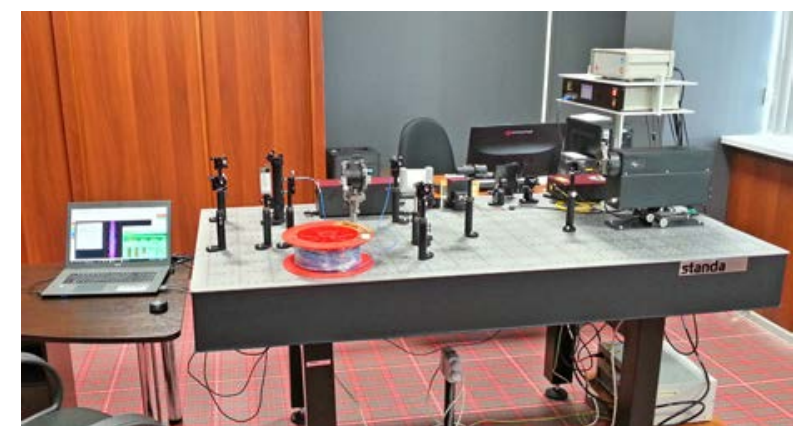

Pис. 2. Внешний вид Государственного вторичного эталона единицы длительности импульса пазерного излучения в диапазоне от $3 \cdot 10^{-12}$ до $1 \cdot 10^{-9} \mathrm{C}$
Таблица 1. Основные характеристики комплекса технических средств для воспроизведения, хранения и передачи единицы длительности импульса лазерного излучения в диапазоне от $5 \cdot 10^{-11}$ до $1 \cdot 10^{-9}$ с в составе Государственного первичного специального эталона ГЭТ 187-2016

\begin{tabular}{|l|l|}
\hline Длина волны, $\lambda_{0}$, мкм & 0,755 \\
\hline Энергия импульса, Q, Дж & $\leq 5 \cdot 10^{-10}$ \\
\hline Длительность импульса, $\tau_{\mathrm{H}}, \mathrm{c}$ & $5 \cdot 10^{-11}-1 \cdot 10^{-9}$ \\
\hline $\begin{array}{l}\text { Среднеквадратическое отклонение, } \\
\mathrm{S \tau}_{\mathrm{H}}, \%\end{array}$ & 1,5 \\
\hline $\begin{array}{l}\text { Неисключенная систематическая } \\
\text { погрешность, } \theta \tau_{\mathrm{H}}, \%\end{array}$ & 3,0 \\
\hline Стандартная неопределенность, $u_{c}(\tau), \%$ & 2,2 \\
\hline
\end{tabular}

первичного эталона. Отличия заключаются в изменении рабочей длины волны в область максимальной чувствительности распространенных типов фотокатодов ЭОК и расширению диапазона измерений длительности импульса в сторону малых значений за счет использования ЭОК с улучшенным временным разрешением. Разработке и исследованию метрологических характеристик эталона посвящена отдельная публикация [7]. Основные характеристики эталона представлены в табл. 2, внешний вид эталона показан на рис. 2.

Эталон, получивший регистрационный № 2.1.ZZA.0101.2017, прошел первичную аттестацию в 2017 году и утвержден приказом Росстандарта № 406 от 05.03.2018.

3. Включение в Государственную поверочную схему (гПС) для СИ энергии, распределения плотности энергии, длительности импульса и длины волны лазерного излучения в диапазоне длин волн от 0,3

Таблица 2. Основные характеристики Государственного вторичного эталона единицы длительности импульса лазерного излучения в диапазоне от $3 \cdot 10^{-12}$ до $1 \cdot 10^{-9} \mathrm{C}$

\begin{tabular}{|l|l|}
\hline Длина волны, $\lambda_{0}$, мкм & 0,525 \\
\hline Энергия импульса, Q, Дж & $\leq 2 \cdot 10^{-8}$ \\
\hline Длительность импульса, $\tau_{\mathrm{H}}, \mathrm{C}$ & $3 \cdot 10^{-12}-1 \cdot 10^{-9}$ \\
\hline $\begin{array}{l}\text { Среднеквадратическое отклонение, } \\
\mathrm{S \tau}_{\mathrm{H}} \%\end{array}$ & 2,6 \\
\hline $\begin{array}{l}\text { Неисключенная систематическая } \\
\text { погрешность, } \theta \tau_{\mathrm{H}}, \%\end{array}$ & 5,9 \\
\hline $\begin{array}{l}\text { Стандартная неопределенность, } u_{c}(\tau), \% \\
4,0\end{array}$ \\
\hline
\end{tabular}



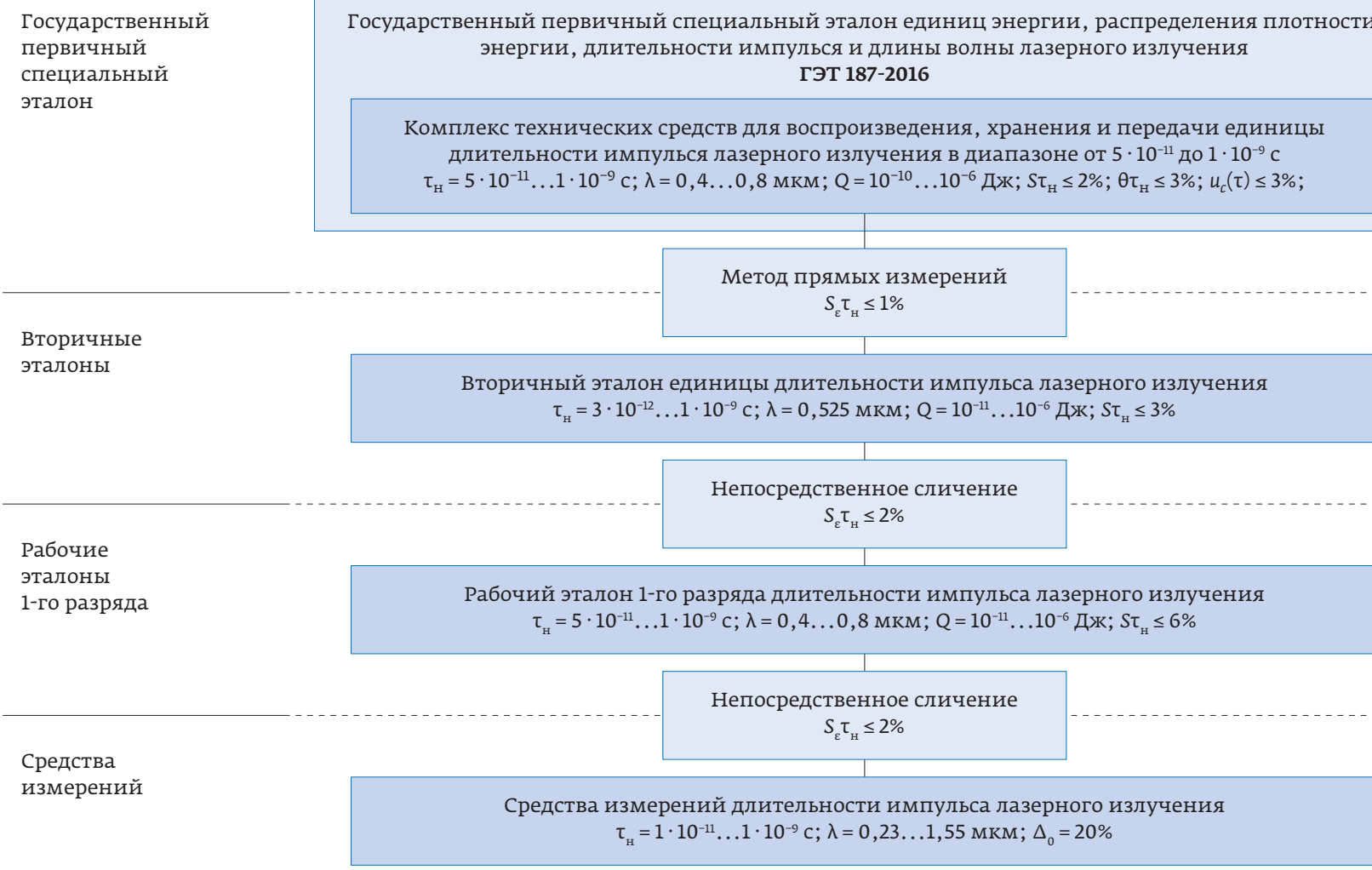

Puс. 3. Новая ветвь дяя СИ длительности импульса лазерного излучения в диапазоне от 10-11 до $10^{-9}$ с в Государственной поверочной схеме

\section{до 2,0 мкм ветви для СИ длительности импульса лазерного излучения в диапазоне от $10^{-11}$ до $10^{-9} \mathrm{C}$}

Действовавшая с 01.07.2013 ГПС для СИ энергии, распределения плотности энергии, длительности импульса и длины волны лазерного излучения в диапазоне длин волн от 0,3 до 2,0 мкм (ГОСТ Р 8.780-2012), возглавляемая Государственным первичным специальным эталоном ГЭТ 187-2010, после введения в эксплуатацию нового комплекса технических средств для воспроизведения, хранения и передачи единицы длительности импульса лазерного излучения в диапазоне от $5 \cdot 10^{-11}$ до $1 \cdot 10^{-9}$ с в составе усовершенствованного эталона ГЭТ 187-2016 нуждалась в обновлении.

В результате в ГПС была включена новая ветвь для СИ длительности импульса лазерного излучения в диапазоне от $10^{-11}$ до $10^{-9}$ с (рис. 3), возглавляемая вновь созданным комплексом технических средств для воспроизведения, хранения и передачи единицы длительности импульса лазерного излучения в диапазоне от $5 \cdot 10^{-11}$ до $1 \cdot 10^{-9}$ с в составе эталона ГЭТ 187-2016.

Обновленная ГПС утверждена приказом Росстандарта № 2088 от 28.09.2018, действует с 01.01.2019.

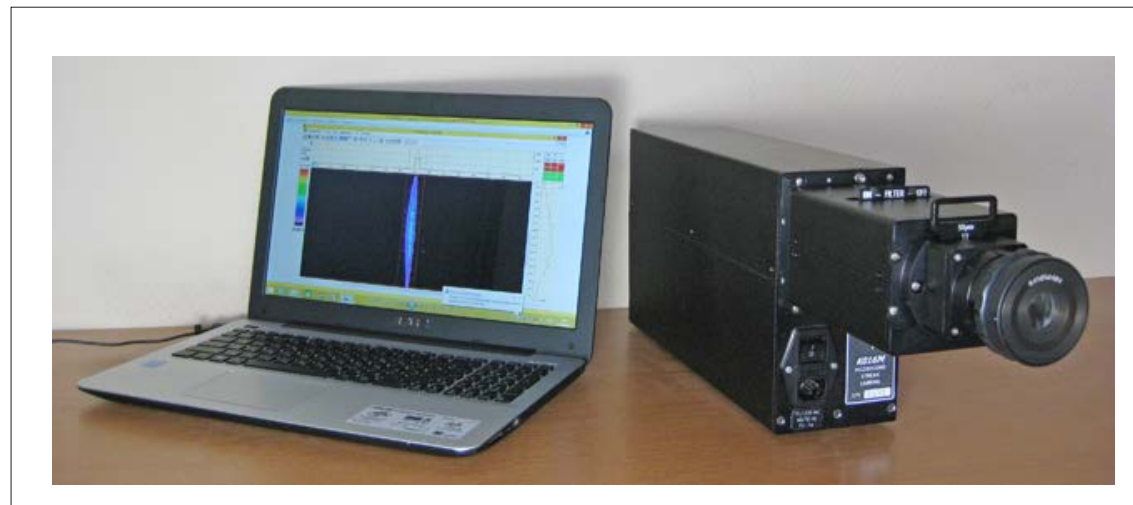

Puc. 4. Внешний вид фотохронографической электронно-оптической камеры к016 вместе с комплектным ноутбуком 
Таблица 3. Метрологические и основные технические характеристики камер К016

\begin{tabular}{|c|c|}
\hline Наименование характеристики & Значение \\
\hline \multicolumn{2}{|l|}{$\begin{array}{l}\text { Предел измерения временных интерва- } \\
\text { лов, нс, не менее: } \\
\text { диапазон развертки, нс/см: }\end{array}$} \\
\hline 0,1 & 0,16 \\
\hline 0,3 & 0,50 \\
\hline 1 & 1,70 \\
\hline 3 & 5,0 \\
\hline 10 & 18,0 \\
\hline $\begin{array}{l}\text { Предел основной погрешности измере- } \\
\text { ния временных интервалов при их дли- } \\
\text { тельности, равной не менее } 2 \text { времен- } \\
\text { ным разрешениям на соответствующем } \\
\text { диапазоне развертки, \% }\end{array}$ & \pm 15 \\
\hline $\begin{array}{l}\text { Область спектральной чувствительно- } \\
\text { сти, нм }\end{array}$ & от 400 до 800 \\
\hline Диапазоны развертки, нс/см & $\begin{array}{l}0,1 \\
0,3 \\
1 \\
3 \\
10\end{array}$ \\
\hline \multicolumn{2}{|l|}{$\begin{array}{l}\text { Временное разрешение при ширине } \\
\text { щели } 50 \text { мкм на длине волны } 532 \text { нм, пс, } \\
\text { не более: } \\
\text { диапазон развертки нс/см: }\end{array}$} \\
\hline 0,1 & 5 \\
\hline 0,3 & 7 \\
\hline 1 & 17 \\
\hline 3 & 50 \\
\hline 10 & 150 \\
\hline $\begin{array}{l}\text { Максимальная частота запуска камеры, } \\
\text { Гц, не менее }\end{array}$ & 8 \\
\hline $\begin{array}{l}\text { Габаритные размеры (без входного объ- } \\
\text { ектива): высота × ширина × длина, мм, } \\
\text { не более }\end{array}$ & $225 \times 115 \times 610$ \\
\hline $\begin{array}{l}\text { Масса (без входного объектива), кг, } \\
\text { не более }\end{array}$ & 10 \\
\hline $\begin{array}{l}\text { Напряжение питания от сети с частотой } \\
\text { 50-60 Гц, В }\end{array}$ & от 85 до 264 \\
\hline $\begin{array}{l}\text { Мощность, потребляемая от питающей } \\
\text { сети, Вт, не более }\end{array}$ & 35 \\
\hline $\begin{array}{l}\text { Условия эксплуатации: } \\
\text { - } \text { температура окружающего воздуха, }{ }^{\circ} \mathrm{C} \\
\text { - относительная влажность воздуха,\%, } \\
\text { не более } \\
\text { - } \text { атмосферное давление, кПа }\end{array}$ & $\begin{array}{l}\text { от +15 до +35 } \\
80 \\
\text { от } 94 \text { до } 106\end{array}$ \\
\hline
\end{tabular}

4. Утверждение типа СИ для фотохронографических электронно-оптических камер К016 как СИ временных параметров импульсного оптического излучения с диапазоном измерений от $1 \cdot 10^{-11}$ до $1,8 \cdot 10^{-8} \mathrm{C}$

Во ВНИИОФИ разработаны и производятся фотохронографические электронно-оптические камеры K016, предназначенные для измерения временных характеристик оптических импульсов. Внешний вид камеры К016 показан на рис. 4.

Камера К016 представляет собой миниатюрную моноблочную конструкцию, в корпусе которой расположены фотохронографический ЭОП с фотокатодом, отклоняющей системой и люминесцентным экраном на ВОП, электронно-оптический усилитель яркости, блоки питания и управления, считывающее устройство на базе ПЗС-камеры. Регистрируемое камерой изображение по интерфейсу USB вводится в компьютер с установленным специальным программным обеспечением (ПО). Основными функциями данного ПО являются обработка и вывод на дисплей изображений, регистрируемых камерой, а также измерение временных характеристик оптических импульсов в соответствии с ГОСТ Р ИСО 11554-2008. ПО осуществляет коррекцию геометрических и фотометрических искажений изображения, возникающих в электронно-оптическом тракте камеры. Поддерживается съемка с накоплением и усреднением, при этом возможна программная коррекция джиттера. Можно выполнять арифметические операции над изображениями, определять различные числовые характеристики изображения, строить профили яркости вдоль пространственной и временной оси, измерять временные характеристики одного или нескольких импульсов. Изображения на дисплее компьютера могут быть представлены в нескольких вариантах псевдоокрашивания, чем достигается увеличение числа визуально различимых уровней яркости. Функция экспорта изображения в графический формат TIFF позволяет при необходимости воспользоваться сторонними средствами обработки изображений. Метрологические и основные технические характеристики камер К016 приведены в табл. 3.

Камеры К016 в 2017 году успешно прошли испытания с целью утверждения типа СИ. Тип СИ утвержден приказом Росстандарта № 1306 от 28.06.2018, сведения внесены в Федеральный информационный фонд по обеспечению единства измерений (регистрационный № 71686-18). Таким образом, камеры К016 могут применяться в сфере государ- 
ственного регулирования обеспечения единства измерений.

\section{ВЫВОДЫ}

Таким образом, в настоящее время у ВНИИОФИ имеется все необходимое для выполнения работ по поверке, калибровке и испытаниям в целях утверждения типа СИ в области измерений временных характеристик импульсного лазерного излучения в пикосекундном диапазоне. Кроме того, ВНИИОФИ может предложить потребителям СИ утвержденного типа для измерения временных характеристик оптических импульсов в пикосекундном диапазоне.

\section{СПИСОК ЛИТЕРАТУРЫ}

1. https://www.keysight.com/ru/pcx-2935671/uxr-series-real-time-infiniiumoscilloscopes.

2. https://www.macom.com/products/product-detail/11000-03.

3. https://www.hamamatsu.com/eu/en/product/type/C11853-01/index.html.

4. Бутслов М. М., Дрожбин Ю.А., Горбенко Б. З., Толмачев А. М., Коринфский Д. Ф., Яковлев В.А., Нефедьев В.А., Ляпин И. В. Измеритепьная техника. 1972; 11: 21-23.

5. Абдрахманов К. Ш., Либерман А.А., Москалюк С.А., Улановский М. В. Измеритепьная техника. 2013; 12: 3-7.

6. Канзюба М. В., Берлизов А. Б., Крутиков В. Н., Фельдман Г. Г. Измерительная техника. 2018;11: 11-14;

7. Kanzyuba M.V., Berlizov A. B., Krutikov V. N., Lebedev V. B., Feldman G. G. Proc. SPIE 10328. 2017;103280G.
Дальнейшие перспективы совершенствования метрологического обеспечения измерений временных характеристик импульсного лазерного излучения в пикосекундном диапазоне связаны с созданием переносных рабочих эталонов, которые позволят осуществлять метрологическую аттестацию си в местах их применения. Пока что передача единицы рабочим СИ производится непосредственно от вторичного эталона, находящегося во ВНИИОФИ. Категория рабочих эталонов, с помощью которых осуществляется передача единицы от вышестоящих эталонов рабочим СИ, уже предусмотрена в действующей c 01.01.2019 ГПС.

\section{REFERENCE}

1. https://www.keysight.com/ru/pcx-2935671/uxr-series-real-time-infiniiumoscilloscopes.

2. https://www.macom.com/products/product-detail/11000-03.

3. https://www.hamamatsu.com/eu/en/product/type/Cl1853-01/index.html.

4. Butslov M. M., Drozhbin Yu.A., Gorbenko B.Z., Tolmachev A. M., Korinfskii D. F., Yakovlev V. A., Nefed'ev V.A., Lyapin I.V. Measurement Techniques. 1972; 15(11):1626-1629

5. Abdrakhmanov K.S., Liberman A.A., Moskalyuk S.A., Ulanovskii M.V. Measurement Techniques. 2014; 56(12):1311-1317.

6. Kanzyuba M.V., Berlizov A. B., Krutikov V. N., Feldman G. G. Measurement Techniques. 2019; 61(11):1052-1056.

7. Kanzyuba M.V., Berlizov A. B., Krutikov V. N., Lebedev V. B., Feldman G. G. Proc. SPIE 10328. 2017;103280G.

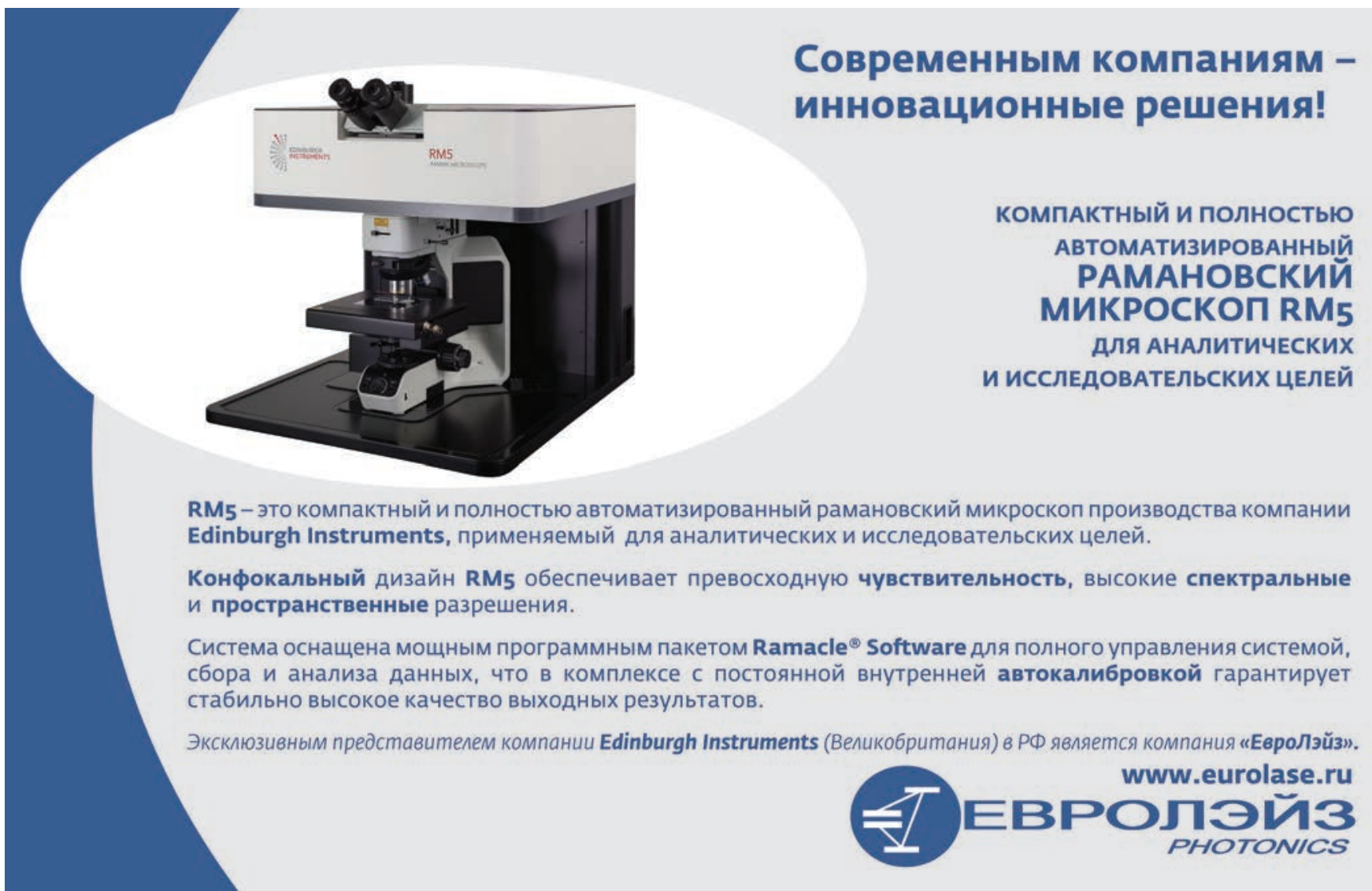

